\title{
The solution of Opioid Flood Problem Based on Big Data Model
}

\author{
Wenhui Cai \\ Zhejiang Yuexiu University of Foreign Languages College of International Finance and Trade
}

Keywords: time series model; infectious disease model; model fitting; cluster analysis; multi-factor influence correlation analysis

Abstract: Aiming at the problem of the drug epidemic in America, we put forward a model of drug epidemic spread. This paper determines the various reasons leading to the current situation, to formulate corresponding effective solutions. This model drew lessons from the spread of the infectious disease model and considering the drug flooding degree affected by time and distance in five states, the drug flood model of the five states is obtained through mathematical analysis. We first make time prediction and then select an appropriate mathematical model for data fitting to determine the parameters. To determine which state and where started the drug epidemic in the first place, we used Python to make a rendering of the drug epidemic degree in each county, so that the drug abuse situation in all counties of the five states could be reflected. When determining the threshold level of drugs, we conducted cluster analysis on drug categories, classified drugs, analyzed charts, determined the drugs with the most significant weight, established the confidence interval and then determined the drug threshold. The model established is used to analyze the influencing factors in combination with the existing policies of the United States to curb the drug epidemic. Some reasonable Suggestions are put forward to curb the drug epidemic.

\section{Introduction}

\subsection{Notations}

Transmission and characteristics of synthetic drugs among the five states

\subsection{Data simplification of the model}

(1) for convenience of calculation, 2010 is the first year;

(2) the data in the table are objective and real, with reliability;

(3) between states, the transmission speed of synthetic drugs is in direct proportion to the distance between the two places. The distance between the five states is measured for mathematical simplification 

$\mathrm{km}$

Ohio -- Kentucky: 411.37 km

Kentucky -- Pennsylvania: 688.91

Pennsylvania -- West Virginia: 445.67 km

Ohio-Pennsylvania: $446.12 \mathrm{~km}$

Ohio -- Virginia: 563.07 km

West Virginia -- Virginia: 332.36 km

Ohio-west Virginia: 297.38 km

Kentucky -- West Virginia: 302.21 $\mathrm{km}$

Kentucky - Virginia: 618.24 km

Pennsylvania -- Virginia: 365.27 km

\section{Assumptions of the model}

(1) the number of people with drug epidemic spreading in unit time is constant $\mathrm{k}$;

(2) the change rate of $\mathrm{S}(\mathrm{t})$ of the number of people prone to take opioids due to various factors is proportional to the product of the number of people in the first category $\mathrm{I}(\mathrm{t})$ and the number of people in the second category $\mathrm{S}(\mathrm{t})$.

(3) the speed of the transition from the first category to the third is proportional to the number of people in the first category.

Symbol specification

$\mathrm{Xi}(0,0)$ : the ratio of drug use at the initial time and place to the population of the state;

$\mathrm{Xi}(\mathrm{x}, \mathrm{t})$ : the distance from the initial $\mathrm{x}$ is the number of drug use time $\mathrm{t}$, and the state's population, the ratio of $x(x, t)=[X 0(x, t)$, the $X 1(x, t), \ldots X m(x, t)], t=0, \ldots$;

$\lambda \mathrm{i}$ : the weight of the ratio of drug use times by county in the year I;

$\operatorname{Bi}(x, t)$ : the distance in the year $\mathrm{I}$ is the proportion affected by the number of propagators at time $\mathrm{t}$ of $\mathrm{x}$;

\section{Drug flooding model in five states}

\subsection{Mathematical analysis}

Under the above assumptions, the following differential equation can be obtained:

$$
\left\{\begin{array}{c}
\frac{d S}{d t}=-r s I \\
\frac{d I}{d t}=r s I-\lambda I \\
\frac{d R}{d t}=\lambda I
\end{array}\right.
$$

Where $\mathrm{r}$ or lambda is the proportionality constant, $\mathrm{r}$ is the rate of drug transmission, or lambda is the ratio controlled by the government.

It is obtained by adding the three equations of equation

$\frac{d}{d t}[S(t)+I(t)+R(t)]=0$

$S(t)+I(t)+R(t)=$ cons $\tan t$

solution:

$$
R(t)=N-S(t)-I(t)
$$

So we have to solve for $\mathrm{S}$ of $\mathrm{t}$ and $\mathrm{I}$ of $\mathrm{t}$ to solve for $\mathrm{R}$ of $\mathrm{t}$. 


$$
\left\{\begin{array}{c}
\frac{d S}{d t}=-r S I \\
\frac{d I}{d t}=r S I-\lambda I
\end{array}\right.
$$

The first and second equations of the system (2.6) are independent of $\mathrm{R}(\mathrm{t})$. Therefore:

$$
\frac{d I}{d S}=\frac{r S I-\lambda I}{-r S I}=-1+\frac{\lambda}{r S}
$$

By the initial condition: when

$$
t=t_{0}, I\left(t_{0}\right)=I_{0}, S\left(t_{0}\right)=S_{0}
$$

Records

$\rho=\frac{\lambda}{r}$

as:

Plugin the above formula to determine the constant

$$
c=I_{0}+S_{0}-\rho \operatorname{In} S_{0}
$$

Solution :

$$
I(S)=-S+\frac{\lambda}{r} \operatorname{In} S+c
$$

$$
I(S)=I_{0}+S_{0}-S+\rho \operatorname{In} \frac{S}{S_{0}}
$$

Next, we discuss the properties of the integral curve (7), which is known from (8)

$$
I^{\prime}(S)=-1+\frac{\rho}{S} \begin{cases}<0 & S>\rho \\ =0 & S=\rho \\ >0 & S<\rho .\end{cases}
$$

So when $S<\rho, I(S)$ is an increasing function of $S$, and $S<\rho, I(S)$ is a minus function of $S$. Moreover, then $I(0)=-\infty, I(S)=I_{0}>0$, from the intermediate value theorem of continuous function and monotonicity, there is a unique point $S_{\infty}, 0<S_{\infty}<S_{0}$, so that $I\left(S_{0}\right)=0$, and when $S_{\infty}<S \leq S_{0}, I(S)>0$.

We know that when $I=0 \quad \frac{d S}{d t}=0, \frac{d I}{d t}=0,{ }_{\text {so }}\left(S_{\infty}, 0\right)$ is the equilibrium point of the system. When $t \geq t_{0}$ the graph of the equation is shown in the figure, as $t$ goes fromt0to infinity, the point $(S(t), I(t))$ moves along the curve and in the direction of $S$ decrease, because of $S(t)$ decreases monotonically with time. So, it $S_{0}$ is less than $\rho$, then $I(t)$ reduces to zero monotonously, and $S(t)$ reduces to $S_{\infty}$ monotonously. So if a small group of addicts $I_{0}$ is dispersed among the population $S_{0}, S_{0}<\rho$, and The drug epidemic is hard to spread.

It can be concluded from the above analysis:

The drug epidemic only spreads when the number of addicts in the population exceeds the threshold $\rho=\frac{\lambda}{r}$. 
The above conclusion is also consistent with common sense. When a state's economic development is relatively backward, the population is crowded, the density is high, the scientific and cultural knowledge is backward, the necessary medical conditions and strict medical supervision system are lacking, the living standard of the people is weak, and the drug epidemic will spread quickly [1-3]. On the contrary, when a state has a relatively developed economy, a low population density, right social conditions, good medical conditions and proper management, and a superior living standard, the spread of drug epidemic in this state is difficult to spread and will soon stop in a limited range [4-6].

\section{Model establishment}

\subsection{Models in PA and WV}

Through the mathematical analysis, it is concluded that the drug flood model for $\frac{d r}{d t}=k^{2} \sec \left(w^{2} * 0.5 k t-m\right)$

.For the spread of the drug epidemic, we predicted the model of the drug epidemic and obtained the initial location through the development model of test times over time of each state and the weight ratio of each county in the state. For the time series model of each state, we use curve to fit [7].

Assuming that the probability of the number of tests in the first year is $P(x, t), P(x, t)$ is a function of the distance between the two places and the time, according to the drug epidemic model, the growth rate of the number of drug tests $P(t)$ is proportional to $P(t)\left(1-\frac{P(t)}{K}\right)$. Where, $K$ is the maximum number of tests a city can achieve, which is the most severe case of the drug epidemic that may occur. When $P(t)$ is very small, $\left(1-\frac{P(t)}{K}\right)$ it is approximately equal to 1 , so the rate of increase in the number of tests is proportional to the continuous increase in $P(t)$. However, when $P(t)$ is more significant than $0.5 K$ and $\left(1-\frac{P(t)}{K}\right)$ is less than 0.5 , the growth rate of test times gradually slows down.

$$
P(0, t)^{2}=0.7436 \sec (493222(0.5 * 0.7436 t-3.104)) \quad(\mathrm{PA})
$$

WV and PA are close to each other, and their models are at similar stages, so the same model can be used to solve the problem.

The value is close to the actual model and conforms to the actual situation. Therefore, we believe that the fitting function can well reflect the real situation. There are

$$
P(0, t)=-0.001314^{2} \sec \left(-0.004142^{2}\left(0.5^{*}-0.001314 t-0.5269\right)\right)
$$

\subsection{Models in KY and VA}

It can be seen from the figure that $\mathrm{OH}$ city is at the initial stage of the drug flooding model. In the future, the drug flooding in $\mathrm{OH}$ city will continue to rise until the critical value is reached. Therefore, $\mathrm{OH}$ city will be the first city to reach the threshold value in the future

Considering the initial ground, the modified equation for $P^{2} \sec (0, t)=k\left(w^{2}(0.5 k t-m)\right)$ Using the known data and Matlab interpolation fitting, get

$$
\mathrm{k}=0.7436 \quad(0.6997,0.7876) \quad \mathrm{w}=0.4932 \quad(0.2573,0.729) \quad \mathrm{m}=3.104 \quad(0.2371,5.971)
$$


Similarly, $k^{2} \sec \left(w^{2}(0.5 k t-m)\right)$

Using the known data and Matlab interpolation fitting, get

$\mathrm{k}=-0.0001314(-0.00156,0.001297) \mathrm{w}=-0.004142(-0.01578,0.00749) \mathrm{m}=0.5269(0.4405,0.6133)$

generally, conform to the development model of the original place.

Set $P(x, t)^{2}=\sec \left(w^{2} k *(0.5 k t-m-(x-x k Y))\right)$

Using the known data and Matlab interpolation fitting, get

$\mathrm{k}=0.7706 \quad(0.756,0.7852) \quad \mathrm{w}=0.5514 \quad(0.4461,0.6567) \quad \mathrm{m}=1.918 \quad(1.586,2.249)$

The value is close to the actual model and conforms to the actual situation. Therefore, we believe that the fitting function can well reflect the real situation. There are

$$
\begin{aligned}
& P^{\prime}(x, t)=P^{\prime}(4, t)=\frac{x}{7}\left(0.7706^{2} * \sec \left(0.5514^{2}(0.5 * 0.7706 t-1.918)\right)\right) \\
& P^{\prime}(x, t)=P^{\prime}(7, t)=\frac{x}{4}\left(0.7706^{2} * \sec \left(0.5514^{2}(0.5 * 0.7706 t-1.918)\right)\right) \\
& (\text { model KY })
\end{aligned}
$$

\subsection{Models in $\mathrm{OH}$}

For $\mathrm{OH}$ state with relatively large policy influence, I modified the original model and designed $P^{\prime}(x, t)=k^{*} t^{*} \sin (w t)+m$ by combining with the autonomy policies of $\mathrm{OH}$ city over the years.

Using the known data and Matlab interpolation fitting, get

$$
\begin{array}{lll}
\mathrm{k}=0.05949 \quad(0.05031,0.06866) & \mathrm{w}=0.2527(0.2189,0.2864) & \mathrm{m}=0.5858(0.5465,0.6252) \\
P^{\prime \prime}(x, t)=0.05949 * t * \sin (0.2527 t)+0.5858 \quad(\mathrm{OH}) &
\end{array}
$$

It can be seen from the figure that $\mathrm{OH}$ city is at the initial stage of the drug flooding model. In the future, the drug flooding in $\mathrm{OH}$ city will continue to rise until the critical value is reached. Therefore, $\mathrm{OH}$ city will be the first city to reach the threshold value in the future

\subsection{Counties analysis}

The intra-state county weight model

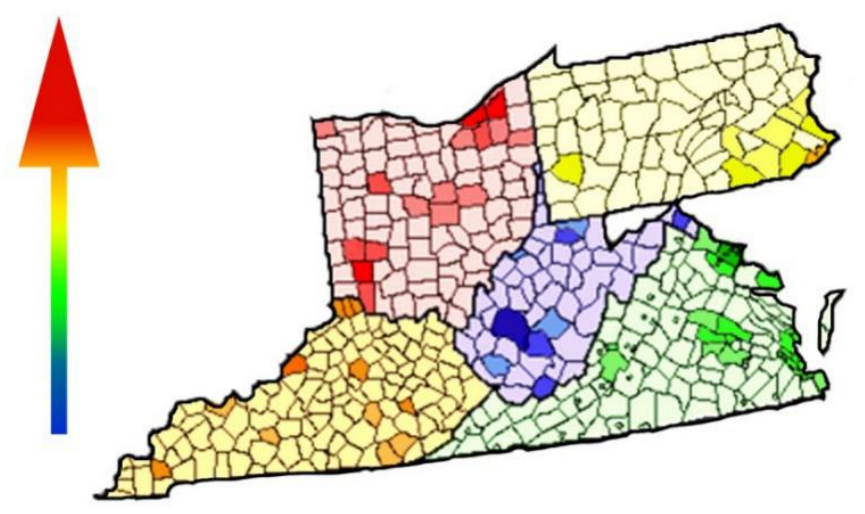

Figure 1: Opioid epidemic

From the above materials, it can be concluded that the PA city was the first state where the drug epidemic occurred. Among PA cities, PHILADELPHIA was the region with the most significant 


\begin{tabular}{|c|c|c|c|c|c|}
\hline \multicolumn{6}{|c|}{ SubstanceName } \\
\hline Heroin & 310315 & Methorphan & 309 & Thebaine & 11 \\
\hline Oxycodone & 110173 & Mitragynine & 196 & Butorphanol & 8 \\
\hline Hydrocodone & 47370 & $\begin{array}{c}\text { Fluoroisobutyryl } \\
\text { fentanyl }\end{array}$ & 155 & $\begin{array}{c}\text { Furanyl/3-Furanyl } \\
\text { fentanyl }\end{array}$ & 7 \\
\hline Fentanyl & 47339 & Benzylfentanyl & 152 & o-Fluorofentanyl & 7 \\
\hline Buprenorphine & 34839 & Meperidine & 104 & p-Fluorofentanyl & 7 \\
\hline Morphine & 10582 & Dextropropoxyphene & 78 & U-51754 & 7 \\
\hline Methadone & 8713 & Phenyl fentanyl & 64 & $\begin{array}{l}\text { Tetrahydrofuran } \\
\text { fentanyl }\end{array}$ & 6 \\
\hline Tramadol & 6399 & Opiates & 60 & 4-Methylfentanyl & 5 \\
\hline Carfentanil & 5828 & Pentazocine & 55 & Acetyldihydrocodeine & 5 \\
\hline Oxymorphone & 5363 & Crotonyl fentanyl & 39 & Isobutyryl fentanyl & 4 \\
\hline Hydromorphone & 5123 & U-48800 & 35 & $\begin{array}{c}\text { 3,4-Methylenedioxy } \\
\text { U-47700 }\end{array}$ & 3 \\
\hline Codeine & 4150 & Opium & 31 & Fluorofentanyl & 3 \\
\hline Furanyl fentanyl & 3219 & $\begin{array}{c}\text { Cyclopropyl/Crotonyl } \\
\text { Fentanyl }\end{array}$ & 26 & Hydrocodeinone & 3 \\
\hline Acetyl fentanyl & 2051 & cis-3-methylfentanyl & 25 & Levorphanol & 2 \\
\hline U-47700 & 1592 & Fluorobutyryl fentanyl & 23 & MT-45 & 2 \\
\hline Acryl fentanyl & 1365 & trans-3-Methylfentanyl & 23 & 3-Fluorofentanyl & 1 \\
\hline 3-Methylfentanyl & 1212 & Valeryl fentanyl & 21 & Alphaprodine & 1 \\
\hline $\begin{array}{l}\text { Cyclopropyl } \\
\text { fentanyl }\end{array}$ & 941 & U-49900 & 17 & Cyclopentyl fentanyl & 1 \\
\hline $\begin{array}{l}\text { 4-Fluoroisobutyryl } \\
\text { fentanyl }\end{array}$ & 698 & $\begin{array}{l}\text { p-Fluorobutyryl } \\
\text { fentanyl }\end{array}$ & 16 & Dihydromorphone & 1 \\
\hline Propoxyphene & 571 & Desmethylprodine & 15 & Metazocine & 1 \\
\hline Butyryl fentanyl & 379 & Pethidine & 14 & Nalbuphine & 1 \\
\hline $\begin{array}{l}\text { Methoxyacetyl } \\
\text { fentanyl }\end{array}$ & 325 & Acetylcodeine & 13 & $\begin{array}{l}\text { p-methoxybutyryl } \\
\text { fentanyl }\end{array}$ & 1 \\
\hline ANPP & $\begin{array}{c}323 \\
\text { Total }\end{array}$ & Dihydrocodeine & 13 & $\begin{array}{l}\text { Remifentanil } \\
\quad 610442\end{array}$ & 1 \\
\hline
\end{tabular}

weight of PA city during 2010-2017. Therefore, PHILADELPHIA in PA city was the first region where the opioid epidemic occurred.

Table 1: Drug survey report

\section{Drug analysis}

When considering drug classification and analyzing data tables, we can use the rough processing of Excel to solve irrelevant variables and determine the total number of occurrences of various drug ingredients [8]-[10]. We can use MATLAB to calculate the confidence interval and obtain the threshold value of drugs. Some of the Heroin.

\section{Influencing factors of drug flooding}

The drug epidemic in the United States is now on the rise. As a social and public problem, the opioid epidemic has its permanence and historicity. Its spread is by no means accidental but closely related to subjective and objective factors in the economy, politics, culture, ideology, and other aspects. The data given by the subject can be summarized as follows: 


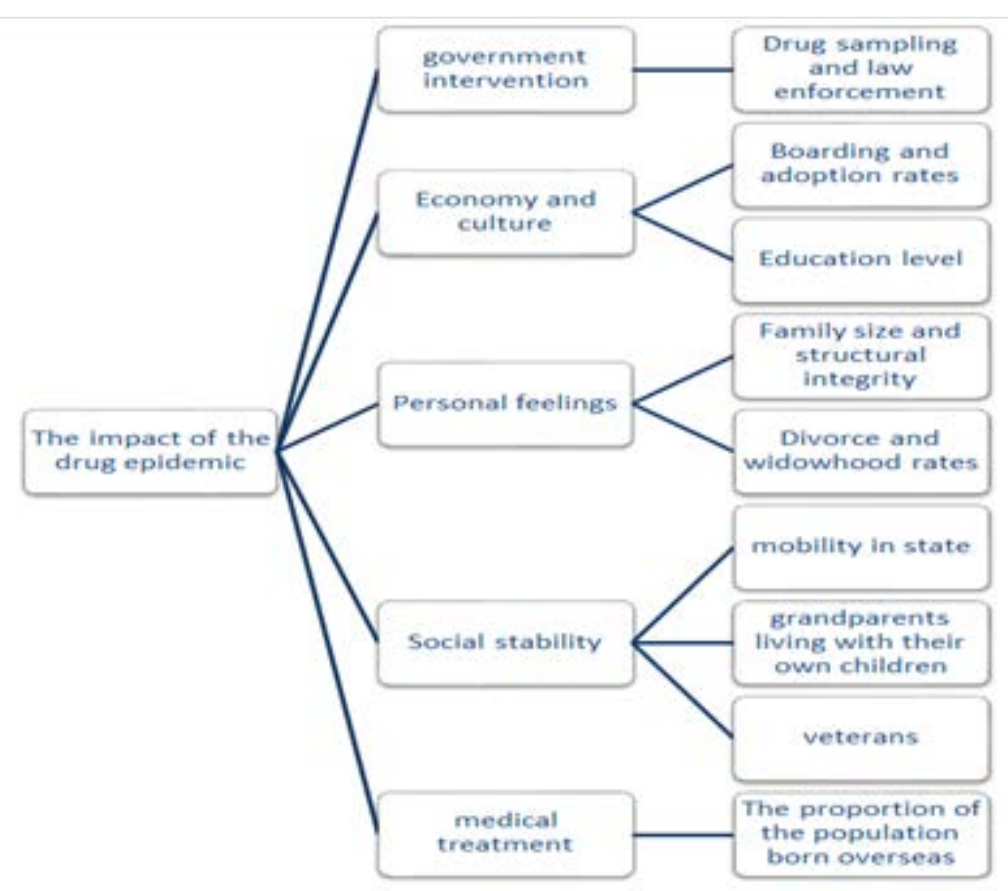

Figure 2: Factors affecting drug proliferation

\subsection{Education level}

The educational level of educatees in each state can reflect the general distribution of industries in each state, which also determines the development trend of industries in each state. People with higher education may also be at risk for opioid abuse, but those with higher education are less likely to abuse opioids than those with lower education.

\subsection{Family size and structural integrity}

It is difficult to maintain the structural stability of a family for a drug addict, and the family will be affected by the size of the effect on the stability of the family structure, the structural integrity of the average family size rate of drug abuse will be the limitation of family and loved ones, to make the drug abuse rate stay at a lower level, full size of the average family structure of the higher the proportion of the total number of states, the lower the level of drug abuse, indicating that the integrity of the family structure in a certain extent, have been drug abuse rate [11]; Overall trends suggest that this may be the reason why overall drug abuse rates continue to rise across the United States [12].

\subsection{Rate of divorce and widowhood}

The divorce and widowed rate increased successively in VA, PA, OH, KY, and WV states, while the overall divorce and widowed rate remained stable.

Although the divorce rate and the widowed rate in each state have remained stable and slightly decreased, the opioid abuse rate in each state is still relatively high, and the opioid abuse rate with a higher divorce rate and the widowed rate is also significantly higher than that in other states. 


\subsection{Mobility in state}

Source of information: extract the demographic survey data of the change of residence within one year in the data table provided, work out the ratio of the change of residence within one year in the state and the change of residence in the total population, and obtain the proportion of the change of residence within the state in the total population.

Chart shows: the chart shows that the population turnover rate increased successively in VA, KY, $\mathrm{WV}, \mathrm{PA}$ and $\mathrm{OH}$ states.

Analysis of corollary: the intra-state population flow accounts for about $98 \%$ of the total population flow, and the intra-state population flow reflects the public security environment of the state to some extent. According to the data, the public security environment of VA, KY, WV, PA, and $\mathrm{OH}$ states gradually declines, which promotes the circulation and illegal abuse of opioids.

\subsection{Grandparents living with their children}

Source of information: the survey data on the change in the number of grandmothers and grandfathers living with children in the provided data table were extracted.

The ratio of KY state to WV state was significantly higher than that of other states, and the ratio increased year by year in all states.

Analysis of corollary: jobs and the economy under pressure, WV and KY states parents choose to send their children to their grandmother and grandfather side to alleviate the burden of family life, but at the same time children without parents' care, more accessible to revolt in the process of the formation of personality, in the long run, this forms source of social instability factors; The chart also shows that the ratio is rising in all the states surveyed, which partly reflects that the overall employment situation is not optimistic and the economic pressure is on the rise.

\subsection{Ratio of veterans}

Source of information: extract the survey data of the number of veterans of citizens over 18 years old in the data table provided, and take the ratio of the number of veterans to the number of citizens over 18 years old.

The ratio of VA, WV, OH, KY, and PA decreased successively, and the proportion of veterans reached the lowest in 2011.

Analysis of corollary: because of the gulf war the U.S. involvement in the last century, and involvement in the Iraq war and the war in Libya at the beginning of the century, not only increased the number of U.S. military war, American soldiers could also experience a war is a significant shift and war brings the psychological shadow, in order to eliminate the shadows, many people turn to drugs and alcohol, tobacco, could have on opioid dependence. Although the ratio of VA, WV, OH, $\mathrm{KY}$ and PA decreased successively, VA states had a better economic foundation and social welfare system, and veterans had better life security. Judging from the degree of opioid abuse in other states, social stability factors in other states might be affected by veterans.

\subsection{Boarding and adoption rates}

Source of information: the survey data of the number of people related to the homeowners in the data table provided are extracted. A part of the population is judged to be the population unrelated to the homeowners. 
From 2010 to 2016, VA state had the highest proportion of this type of population, while OH state had the lowest proportion of this type of population.In 2011 there was a big increase in this type of population.

This type of population is preliminarily judged as homestay population or adoptive population. The number of homestay populations and adoptive populations can directly reflect the economic status of adoptive and homestay families, and the ratio is the highest in VA state.

\section{Strategies to address the opioid crisis}

Government: strengthen regulatory control, control population mobility, care about the living conditions of the people in the slums and the growth of some teenagers who are not under the supervision of their parents, control the unemployment rate, ensure the employment of the people, and improve the national happiness index.

Food and drug administration (FDA); To ensure the safety and effectiveness of medicines, to promote innovation in medicines, and to help the public understand scientifically the medicines they use.

Drug enforcement administration (DEA) : as the regulatory agency for the enforcement of drug laws and regulations in the United States, DEA integrates administration and justice, publicizes to the public the impact of the use of illegal drugs, misuse of prescription drugs and drug abuse on health, and establishes long-term cooperation and collaboration with drug manufacturers, dealers and users to fight against drug traffickers.

\section{Analysis of model results:}

The model established is the drug epidemic transmission model in five states, which reflects the process from the drug epidemic in one state to drug epidemic suppression. Each state will experience the emergence and aggravation of drug epidemic, and then reach the threshold and maintain it for a period of time, until the government realizes the problems caused by drug epidemic and takes regulatory measures, and at the later stage, the drug epidemic phenomenon will be suppressed, and the curve will decline year by year, at which time the drug epidemic will reach a controllable level.

The analysis to the contents of the chart, select the factors related to the first question, reasonable analysis every factor change over time and the differences, combined with the first asking drug flood model, flood analysis of drugs and the correlation of various factors, in combination with access to the data, data rationality of each factor can be determined, and then correlation analysis, it is concluded that the main influence factors and secondary influence factors.

\section{Sensitivity analysis:}

Since many factors are influencing the drug epidemic in this problem, and the randomness is significant, we carried out correlation analysis for multiple factors. The sensitivity of the drug flooding model to these parameters reflects the significance of various factors influencing drug flooding. On the contrary, through the analysis of various influencing factors of the model, the actual rationality of the model can be reflected and tested.

However, we found that this problem was caused by multiple factors in a complicated situation, and its indicators mainly included population mobility, marriage and family stability, number of ex-servicemen, government control and other factors, so it was particularly important to analyze the correlation effect of various factors on the results.

Evaluation and improvement of the model 


\section{Conclusions}

\subsection{Advantages of the model}

(1) Drawing on the existing infectious disease model, the model of drug flooding degree was put forward after optimization and improvement. Considering the different stages of experience in different states, the extent of drug flooding varying with time and distance is proposed.

(2) Analyze several factors, consider the rationality of influencing factors with the model, and select the primary factors and secondary factors.

(3) When analyzing the model and influencing factors, the factual rationality of the results is guaranteed by consulting the data.

\subsection{Disadvantages of the model}

(1) When dealing with the influencing factors, data processing is inadequate to deal with some minor cases, resulting in certain errors in the results.

(2) When giving measures to curb drug abuse, we should consider the situation throughout the United States. Because of the greater autonomy of each state in the United States, the degree of development between different states, and government policies, there may be differences, which leads to the suggestion that different states should be analyzed according to different state conditions.

\subsection{Improvement of the model}

Because of the shortcomings of the model, we believe that in the analysis of the influencing factors of the model, we should first analyze the actual situation, understand the influencing factors and then conduct data analysis, so that the analysis data will not miss some influencing factors.

\section{References}

[1] Shafer S L, Flood P. The Pharmacology of Opioids, Geriatric Anesthesiology. 2008.

[2]Z. Zhao, J. Wang and Y. Liu, "User Electricity Behavior Analysis Based on K-Means Plus Clustering Algorithm," 2017 International Conference on Computer Technology, Electronics and Communication (ICCTEC), Dalian, China, 2017, pp. 484-487. doi: 10.1109/ICCTEC.2017.00111

[3] Flood J F, Garland J S, Morley J E. Evidence that cholecystokinin-enhanced retention is mediated by changes in opioid activity in the amygdala, Brain Research, 1992, 585(1-2):94.

[4] Morley J E, Flood J F, Silver A J. Opioid peptides and aging, Annals of the New York Academy of Sciences, 2010, 579(1):123-132.

[5] L. Yi and W. Yi, "Decision Tree Model in the Diagnosis of Breast Cancer," 2017 International Conference on Computer Technology, Electronics and Communication (ICCTEC), Dalian, China, 2017, pp. 176-179. doi: 10.1109/ICCTEC.2017.00046

[6]Zhu C J, Wu L P, Li S. Flood Forecasting Research Based on the Chaotic BP Neural Network Model, Key Engineering Materials, 2010, 439-440:411-416.

[7] Y. Wu, Y. Liu, S. H. Ahmed, J. Peng and A. A. A. El-Latif, "Dominant Dataset Selection Algorithms for Electricity Consumption Time-Series Data Analysis Based on Affine Transformation," in IEEE Internet of Things Journal. doi: 10.1109/JIOT.2019.2946753

[8] Marouane E M, Mostafa E, Mohammad E. Toward an optimal model based on inequality measures for treatment of historical \& real time flood's dataset, Information Science \& Technology. 2015. 
[9] Zhang L, Zhuo Q. Notice of RetractionThe Research on Problem of Flood Insurance Pricing Based on the DFA in P. R. China,/International Conference on Management \& Service Science. 2010.

[10] Bannwarth B. Will Abuse-Deterrent Formulations of Opioid Analgesics Be Successful in Achieving Their Purpose? Drugs, 2012, 72(13):1713-1723.

[11] Lankenau S E, Walley A. Opioids and deaths, New England Journal of Medicine, 2011, 364(7):686.

[12] Racz G B, Noe C, Hansen H, et al. Medicolegal Aspects of Pain Medicine with Special Reference to Opioid Therapy, Techniques of Neurolysis. 2016. 